\title{
Germline whole exome sequencing of a family with appendiceal mucinous tumours presenting with pseudomyxoma peritonei
}

\author{
Mei Sim Lung1', Catherine A. Mitchell², Maria A. Doyle, Andrew C. Lynch, Kylie L. Gorringe,
}

David D. L. Bowtell ${ }^{6,7,8}$, Australian Ovarian Cancer Study Group, lan G. Campbell ${ }^{1,2,7^{*}+}$ (i) and Alison H. Trainer ${ }^{1,7,9,10 \dagger}$

\begin{abstract}
Background: Familial cases of appendiceal mucinous tumours (AMTs) are extremely rare and the underlying genetic aetiology uncertain. We identified potential predisposing germline genetic variants in a father and daughter with AMTs presenting with pseudomyxoma peritonei (PMP) and correlated these with regions of loss of heterozygosity $(\mathrm{LOH})$ in the tumours.

Methods: Through germline whole exome sequencing, we identified novel heterozygous loss-of-function (LoF) (i.e. nonsense, frameshift and essential splice site mutations) and missense variants shared between father and daughter, and validated all LoF variants, and missense variants with a Combined Annotation Dependent Depletion (CADD) scaled score of $\geq 10$. Genome-wide copy number analysis was performed on tumour tissue from both individuals to identify regions of $\mathrm{LOH}$.

Results: Fifteen novel variants in 15 genes were shared by the father and daughter, including a nonsense mutation in REEP5. None of these germline variants were located in tumour regions of $\mathrm{LOH}$ shared by the father and daughter. Four genes (EXOG, RANBP2, RANBP6 and TNFRSF1B) harboured missense variants that fell in a region of $\mathrm{LOH}$ in the tumour from the father only, but none showed somatic loss of the wild type allele in the tumour. The REEP5 gene was sequenced in 23 individuals with presumed sporadic AMTs or PMP; no LoF or rare missense germline variants were identified.
\end{abstract}

Conclusion: Germline exome sequencing of a father and daughter with AMTs identified novel candidate predisposing genes. Further studies are required to clarify the role of these genes in familial AMTs.

Keywords: Pseudomyxoma peritonei, Appendiceal tumour, Familial, Germline predisposition, Exome sequencing

\footnotetext{
* Correspondence: ian.campbell@petermac.org

${ }^{\dagger}$ lan G. Campbell and Alison H. Trainer are co-senior authors

${ }^{1}$ Research Division, Cancer Genetics Laboratory, Peter MacCallum Cancer

Centre, Victorian Comprehensive Cancer Centre Building, 305 Grattan St., Melbourne, Victoria VIC 3000, Australia

${ }^{2}$ Department of Pathology, Peter MacCallum Cancer Centre, Melbourne, Victoria, Australia

Full list of author information is available at the end of the article
}

(c) The Author(s). 2020 Open Access This article is licensed under a Creative Commons Attribution 4.0 International License, which permits use, sharing, adaptation, distribution and reproduction in any medium or format, as long as you give appropriate credit to the original author(s) and the source, provide a link to the Creative Commons licence, and indicate if changes were made. The images or other third party material in this article are included in the article's Creative Commons licence, unless indicated otherwise in a credit line to the material. If material is not included in the article's Creative Commons licence and your intended use is not permitted by statutory regulation or exceeds the permitted use, you will need to obtain permission directly from the copyright holder. To view a copy of this licence, visit http://creativecommons.org/licenses/by/4.0/. The Creative Commons Public Domain Dedication waiver (http://creativecommons.org/publicdomain/zero/1.0/) applies to the data made available in this article, unless otherwise stated in a credit line to the data. 


\section{Background}

Appendiceal mucinous tumours (AMTs) are rare, occurring at an age-adjusted incidence of 0.12 per million individuals, with a median age at diagnosis of 59 years and no gender bias [1]. Pseudomyxoma peritonei (PMP) is a clinical term describing gelatinous ascites, associated with the presence of mucin-producing cells within the peritoneal cavity, usually associated with an appendiceal mucinous neoplasm, either a low-grade appendiceal mucinous neoplasm (LAMN) or a mucinous

adenocarcinoma [2]. Whether PMP occurs in all, or just a molecular subset of AMTs is unknown.

Molecular studies of PMP and AMTs are limited, but somatic mutations have been identified in KRAS (60$100 \%$ of sequenced cases), GNAS (30-74\%), SMAD4 (16\%), and TP53 (5-14\%) [3-9]. Compared to colorectal cancer, somatic mutations in the $A P C$ gene are observed at a much lower frequency, ranging between 0 and $33 \%$ $[4,7,9,10]$. An RNF43 stop-gained mutation in a region of $\mathrm{LOH}$ in a LAMN has recently been described [10]. In 5 LAMNs, a mutation signature consistent with deamination of 5-methylcytosine was commonly detected [10].

Familial forms of AMTs are rare, with only two reported cases in the literature. The first family comprised monozygotic twin brothers [11]. The first twin was diagnosed with PMP at the age of 35 during an umbilical hernia repair, and he was subsequently found to have a perforated AMT. After this diagnosis, his asymptomatic twin underwent a prophylactic appendectomy which identified a non-perforated AMT. Somatic LOH of the APC locus was identified in the second AMT but not in the original case. No germline $A P C$ mutation data were available.

The second family comprised a brother and sister diagnosed with AMTs at the ages of 69 and 77 years respectively [12]. The brother presented with acute appendicitis and an AMT was identified at surgery, while his sister presented with increasing abdominal girth and was found to have PMP and an AMT. An assessment for Lynch syndrome was performed in this family. The sister's AMT had normal immunohistochemistry staining for the mismatch repair proteins MLH1, MSH2, MSH6 and PMS2, and her tumour was microsatellite stable, whilst her brother underwent constitutional mutation analysis of $M L H 1, M S H 2$ and MSH6 which did not identify a pathogenic variant.

Here, we report the first familial parent-child PMP case, a father (P1) and daughter (P2) who were both diagnosed with PMP secondary to an AMT at the ages of 66 and 51 years respectively (Fig. 1). P1 was incidentally found to have PMP on staging CT for a Gleason 6 prostate cancer. A diagnosis of PMP was made on diagnostic laparotomy and he underwent drainage of a large abdominal cyst. Subsequent cytoreductive surgery undertaken 4 years later when he became symptomatic, identified a ruptured LAMN. His daughter (P2) presented with 6 months of menorrhagia, dysmenorhoea and pelvic and right upper quadrant discomfort. She was found to have PMP and a moderately differentiated mucinous adenocarcinoma of the appendix.

Identifying the germline genetic aetiology of rare familial colorectal cancer syndromes such as familial adenomatous polyposis has led to a better understanding of many somatic pathways and mechanisms underlying sporadic forms of the disease. Thus, we sought to identify predisposing genes in this family through a germline whole exome sequencing approach and looked for regions of $\mathrm{LOH}$ in their tumour tissues as a secondary filter for potentially pathogenic variants.

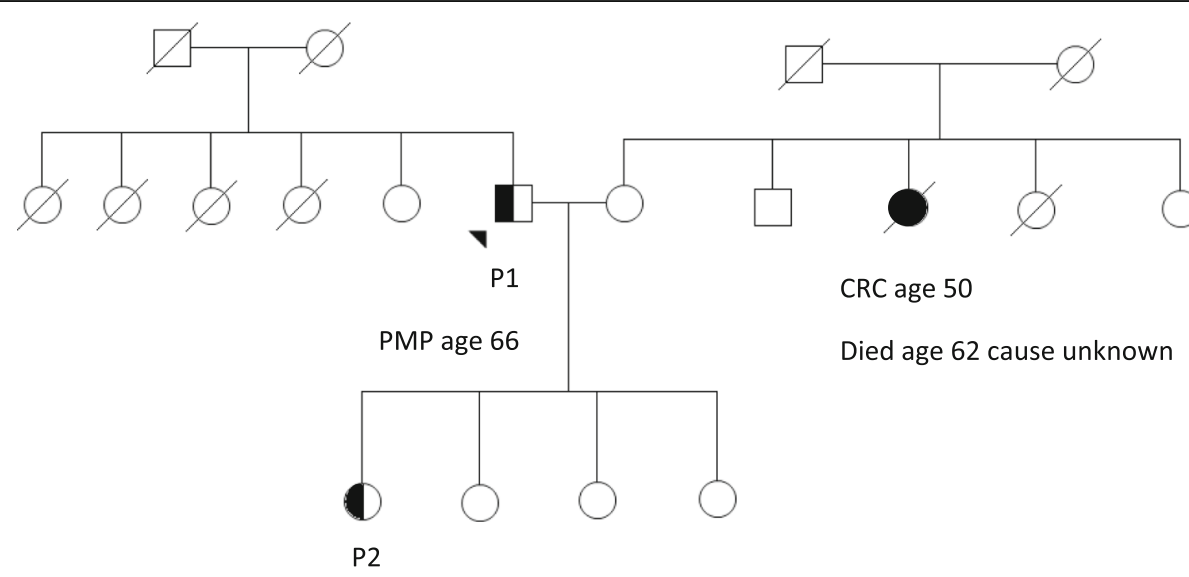

PMP age 51

Fig. 1 Pedigree of parent child pseudomyxoma peritonei (PMP). Legend. The father (P1) presented with PMP at age 66, while his daughter (P2) developed PMP at age 51 


\section{Methods}

\section{Ethics}

All procedures performed involving human participants were in accordance with the ethical standards of the Peter MacCallum Cancer Centre Human Research and Ethics Committee (project number 10_83) and with the 1964 Helsinki declaration and its later amendments or comparable ethical standards. Written informed consent was obtained from all individual participants included in the study. P1 and P2 signed patient information and consent forms to participate in this study, which included the consent for publication of de-identified data.

\section{Whole exome sequencing}

One $\mu \mathrm{g}$ of germline DNA was obtained from peripheral leucocytes and fragmented using the Covaris S2 System (Covaris, Woburn, MA, USA). The SureSelect Human All Exon v1 (Agilent, Santa Clara, CA, USA) was used for exome enrichment according to the manufacturer's protocol. Paired-end 100 base pair reads were sequenced on a HiSeq2000 (Illumina Inc., San Diego, CA, USA) instrument. Both exomes passed sequencing quality control with mean target base coverages of $129 x$ and $121 x$ for P1 and P2 respectively and $>95 \%$ of targeted bases covered more than $10 x$.

\section{Sequence alignment and variant calling}

Raw sequence reads were quality checked with FastQC [13] and trimmed for low quality bases and adaptor if necessary using Cutadapt [14]. Reads were aligned to the human genome (GRCh37 assembly) using BWA-MEM [15]. Duplicate reads were marked using Picard [16] followed by merging of BAM files for both individuals. Local realignment around indels was performed on the merged BAM files using the Genome Analysis Tool Kit (GATK) software v3.1 [17]. Subsequently, base quality score recalibration was performed using GATK software. Single nucleotide variants (SNVs) and indels were identified using the GATK HaplotypeCaller and annotated with information from Ensembl release 73 using Ensembl's Perl API and Variant Effect Predictor [18, 19]. Each variant was annotated with its frequency in the 1000 Genomes Project [20], the National Heart, Lung and Blood Institute (NHLBI) Grand Opportunity (GO) Exome Sequencing Project [21] and an in-house exome dataset of 147 familial breast cancer cases [22]. The likely pathogenic consequence for each variant was determined by Polyphen [23], SIFT [24], and Combined Annotation Dependent Depletion (CADD) scaled score [25].

\section{Exome data analysis}

For genes with multiple transcripts, transcripts were prioritised on 1) most to least deleterious predicted impact of variant on protein function (Supplementary Data 1 and
2) RefSeq transcript. The highest ranking transcript was taken forward for further analysis. LoF variants and missense variants which met the following criteria were considered for further analysis: [1] Phred variant quality score of $>30$, and [2] variant allele frequency between 0.15 and 0.8 . For identification of novel variants shared between P1 and P2, variants were excluded if they were present in control cohorts: 1000 Genomes Project, NHLBI GO Exome Sequencing Project or an in-house cohort of 147 Australian familial breast cancer exomes. All loss-offunction (LoF) variants (truncating frameshift, nonsense, essential splice site), and missense variants with a CADD scaled score $\geq 10$ were manually checked in the Integrated Genome Viewer (IGV) [26, 27].

Variants shared between P1 and P2 which were confirmed on Sanger sequencing were checked in the Genome Aggregation Database v2 dataset (gnomAD) [28], comprising exome and genome data from 125,748 and 15, 708 unrelated individuals respectively, for the population frequency, to confirm that these variants were rare or novel. Variants with a frequency greater than $1 \mathrm{X} 10^{-4}$ in the gnomAD dataset were considered too common to account for the development of AMTs and were excluded.

\section{Whole genome amplification and Sanger sequencing} Candidate variants were confirmed by Sanger sequencing using whole-genome amplified DNA from P2. Whole-genome amplification of genomic DNA was performed using the REPLI-g Midi Kit (Qiagen, Redwood City, CA, USA). PCR primers were designed using the Primer3 program v0.4.0 $[29,30]$ and are listed in Supplementary Data 2. DNA fragments were amplified using HotStarTaq DNA Polymerase (Qiagen, Redwood City, CA, USA), purified using ExoSAP-IT PCR Purification Kit (USB Corporation, Cleveland, OH, USA), and sequenced using the Big Dye Terminator v3.1 kit (Applied Biosystems, Foster City, CA, USA). Sanger sequencing was performed on an ABI3130 Sequencer (Applied Biosystems), and visualised in Geneious 5.6.2 software (BioMatters Ltd., Auckland, New Zealand).

\section{Tumour micro-dissection and analysis}

Both tumours were reviewed by a clinical pathologist with expertise in this area. Consecutive $10 \mu \mathrm{m}$ sections were cut from the formalin fixed paraffin embedded PMP specimens with the highest tumour content, and stained with haematoxylin and eosin. Tumour cells were micro-dissected manually using a 23 gauge needle and somatic DNA was extracted using the DNeasy Blood and Tissue Kit (Qiagen, Redwood City, CA, USA). Somatic copy number analysis of tumours was assayed using the OncoScan Molecular Inversion Probe assay (Affymetrix, Santa Clara, CA, USA) on 50-75 ng of somatic DNA, and the data analysed using Nexus Copy 
Number ${ }^{\text {tw }}$ software (Biodiscovery, Inc., El Segundo, CA, USA). There was no matched control copy number data available for P2. The Oncoscan molecular assay comprises $>220,000$ single nucleotide polymorphisms and provides copy number resolution of around $50-100 \mathrm{~kb}$.

To assess if a variant showed somatic LOH, Sanger sequencing was performed using unamplified tumour DNA extracted from the AMT (primers listed in Supplementary Data 3).

\section{Sanger sequencing of candidate genes in an AMT/PMP validation cohort}

Germline DNA from individuals with AMTs or PMP was obtained from the Victorian Cancer Biobank (VCB), the Australian Ovarian Cancer Study (AOCS) and Southampton, UK [31]. Clinical details were extracted from de-identified histopathology reports. Histopathology reports for all PMP samples in the validation cohort were examined to ensure that they were not metastases of known ovarian origin.

Sanger sequencing of all exons of REEP5 was performed using germline DNA from individuals from the PMP validation cohort, using the same methods as described earlier. PCR primers for each exon were designed to include 40 base pairs flanking the intron-exon boundary of each exon (Supplementary Data 4).

\section{Results}

\section{Analysis of genes associated with known familial} colorectal cancer syndromes

Prior to analysis for novel shared germline variants, a targeted analysis was undertaken of 17 genes associated with an increased familial colorectal cancer risk: $A P C$, MUTYH, MLH1, MSH2, MSH6, PMS2, BMPR1A, SMAD4, STK11, EPCAM [32], GREM1, POLE [33], POLD1 [33], BUB1 [34], BUB1B [35], BUB3 [34] and NTHL1 [36]. All these genes excluding STK11 were sequenced with a mean coverage of $>68 \mathrm{X}$ (Supplementary Data 5). No LoF or known pathogenic missense variants were identified in either case.

\section{Identification of shared germline variants}

LoF and missense variants with an allele frequency between 0.15 and 0.8 were selected in order to identify heterozygous variants. A total of 4893 and 4973 variants were identified in P1 and P2, respectively. After excluding any variant previously reported in the 1000 Genomes Project or the NHLBI GO Exome Sequencing Project, or a local cohort of 147 familial breast cancer exomes, a total of 106 and 110 variants remained in P1 and P2, respectively. Of these, 40 variants ( $8 \mathrm{LoF}$ and 32 missense) were shared between P1 and P2. Manual curation of the variant reads in IGV eliminated a further 9 variants as likely artefacts due to misalignment, known hypervariable genes or variants located in areas with low mapping quality. Missense variants were prioritised on their CADD score. A CADD scaled score of $\geq 10$ was used as an inclusion threshold, as this identifies the top $10 \%$ most deleterious substitutions in the human genome [25]. This approach eliminated a further seven missense variants. Following validation by Sanger sequencing and verification of allele frequencies in the gnomAD dataset, the final list of shared variants in 15 genes comprised 1 LoF and 14 missense variants (Table 1). The number of

Table 1 Fifteen validated variants seen in both P1 and P2

\begin{tabular}{|c|c|c|c|c|c|c|}
\hline Gene & Ref_Seq mRNA transcript & cDNA variant & Amino Acid Change & Variant type & Scaled CADD score & variant frequency in gnomAD v2 \\
\hline REEP5 & NM_005669.4 & c. $159 \mathrm{~T}>\mathrm{G}$ & p.Tyr53* & Nonsense & 31 & 0 \\
\hline RHBDL2 & NM_017821.3 & c. $395 C>$ G & p.Gly132Ala & Missense & 29.8 & 0 \\
\hline FGFR4 & NM_022963.2 & c.1988G > A & p.Arg663Gln & Missense & 21.9 & 4.42E-05 \\
\hline CNTN2 & NM_005076.3 & c. $2263 \mathrm{~A}>\mathrm{T}$ & p.Ser755Cys & Missense & 21.2 & $3.98 \mathrm{E}-06$ \\
\hline RANBP2 & NM_006267.4 & c.3683G $>\mathrm{T}$ & p.Gly1228Val & Missense & 19.8 & 0 \\
\hline ZNF747 & NM_023931.2 & c. $254 C>T$ & p.Gly85Glu & Missense & 16.28 & 5.70E-06 \\
\hline EXOG & NM_005107.3 & c. $178 \mathrm{G}>\mathrm{A}$ & p.Ala60Thr & Missense & 16.17 & 0 \\
\hline BRINP3 & NM_199051.1 & C.809A > C & p.Glu270Ala & Missense & 16.1 & 1.60E-05 \\
\hline ASIC1 & NM_020039.3 & c. $463 C>T$ & p.Arg155Cys & Missense & 16.05 & 0 \\
\hline TNFRSF1B & NM_001066.2 & c.1337A > G & p.Glu446Gly & Missense & 15.46 & 3.99E-06 \\
\hline RANBP6 & NM_001243202.1 & c. $251 \mathrm{~A}>\mathrm{C}$ & p.Glu84Ala & Missense & 14.87 & 3.98-E06 \\
\hline$L S R$ & NM_205834.3 & c. $725 C>T$ & p.Thr242lle & Missense & 14.79 & $3.20 \mathrm{E}-05$ \\
\hline MTERFD3 & NM_001033050.1 & c. $607 \mathrm{C}>\mathrm{T}$ & p.Ala203Thr & Missense & 12.04 & 1.20E-05 \\
\hline PAX1 & NM_001257096.1 & c.1379G > T & p.Arg460Leu & Missense & 11.57 & $5.11 \mathrm{E}-05$ \\
\hline$E G F R$ & NM_005228.3 & C.1915A > C & p.ASN639His & Missense & 10.51 & 0 \\
\hline
\end{tabular}


variants remaining after each filtering step is summarised in Supplementary Data 6.

\section{Identification of germline variants associated with concomitant areas of tumour LOH}

Assuming a possible two-hit model for germline tumour suppressor inactivation, analysis was performed to identify germline candidate variants present in regions of $\mathrm{LOH}$ in the tumours.

The Oncoscan array detected somatic LOH (copy number neutral and loss) in $20.3 \%$ and $19.8 \%$ of the tumour genomes of $\mathrm{P} 1$ and $\mathrm{P} 2$, respectively (Tables 2 and 3). Only one region of $\mathrm{LOH}$, a $1.77 \mathrm{Mb}$ region on chromosome 2q, was common to both tumours (Fig. 2). This region contains 33 genes but none harboured likely pathogenic germline variants common to both individuals.

As LOH is not the only mechanism by which a somatic wild type allele can be abrogated, the analysis was extended to incorporate all regions of somatic $\mathrm{LOH}$ present in either P1's or P2's tumour. Missense variants in four genes, EXOG, RANBP2, RANBP6 and TNFRSF1B, were identified in areas of $\mathrm{LOH}$ seen in the tumour from $\mathrm{P} 1$. The $\mathrm{LOH}$ regions in the tumour from P2 did not harbour any shared germline variants.

The variants in EXOG, RANBP2, RANBP6 and TNFRSF1B were sequenced in the tumour DNA of P1 to assess which allele of the gene was lost. RANBP2 showed somatic loss of the mutant allele. Unexpectedly, somatic sequencing of EXOG, RANBP6 and TNFRSF1B demonstrated the presence of both the wild type and mutant alleles (Supplementary Data 7 and 8). These discordant results may relate to the genomic heterogeneity within subclones of the PMP tumours. The DNA used as template for Sanger sequencing was extracted from the primary appendiceal tumour whilst the template DNA for the Oncoscan array was extracted from secondary peritoneal tumour in order to obtain sufficient DNA. Due to the sparse nature of the tumour cells, no remaining DNA was available for Sanger sequencing from the secondary peritoneal tumour.

\section{Screening of the REEP5 gene in a validation cohort of individuals with presumed sporadic AMTs/PMP using Sanger sequencing}

Having identified a LOF variant in REEP5 present in both $\mathrm{P} 1$ and P2, further independent evidence implicating this gene in AMT predisposition was found in a previous case report [37] of an individual with a chromosomal translocation $\mathrm{t}(5 ; 8)(\mathrm{q} 22 ; \mathrm{p} 23.1)$ who was diagnosed with a mucin-secreting appendiceal carcinoma and familial adenomatous polyposis (FAP) at the age of 26 years. Fluorescent in situ hybridisation studies in this case identified a microdeletion encompassing
Table 2 Regions of loss of heterozygosity ( $\mathrm{LOH}$ ) and copy number (CN) loss in pseudomyxoma peritonei tumour from $\mathrm{P} 1$

\begin{tabular}{|c|c|c|c|}
\hline Chromosome & Start & End & Event \\
\hline 1 & 0 & $26,948,921$ & CN Loss/LOH \\
\hline 1 & $147,134,028$ & $147,825,662$ & $\mathrm{LOH}$ \\
\hline 1 & $190,881,536$ & $191,887,248$ & $\mathrm{LOH}$ \\
\hline 2 & $50,914,938$ & $51,446,707$ & $\mathrm{LOH}$ \\
\hline 2 & $99,377,108$ & $243,199,373$ & $\mathrm{LOH}$ \\
\hline 3 & 0 & $48,040,095$ & $\mathrm{LOH}$ \\
\hline 3 & $50,384,337$ & $52,768,237$ & $\mathrm{LOH}$ \\
\hline 3 & $191,022,128$ & $191,856,841$ & $\mathrm{LOH}$ \\
\hline 4 & $21,562,094$ & $22,526,745$ & $\mathrm{LOH}$ \\
\hline 6 & $33,506,076$ & $171,115,067$ & CN Loss/LOH \\
\hline 7 & $22,818,202$ & $23,530,679$ & $\mathrm{LOH}$ \\
\hline 9 & 0 & $33,946,637$ & $\mathrm{LOH}$ \\
\hline 9 & $118,867,206$ & $119,530,524$ & $\mathrm{LOH}$ \\
\hline 10 & $62,401,757$ & $63,554,609$ & $\mathrm{LOH}$ \\
\hline 10 & $69,912,047$ & $70,990,019$ & $\mathrm{LOH}$ \\
\hline 11 & $5,529,179$ & $6,091,608$ & $\mathrm{LOH}$ \\
\hline 11 & $34,356,991$ & $35,043,999$ & $\mathrm{LOH}$ \\
\hline 12 & $21,011,988$ & $22,928,787$ & $\mathrm{LOH}$ \\
\hline 12 & $29,116,987$ & $29,816,788$ & $\mathrm{LOH}$ \\
\hline 12 & $111,166,777$ & $112,632,998$ & $\mathrm{LOH}$ \\
\hline 14 & $36,158,966$ & $36,786,121$ & $\mathrm{LOH}$ \\
\hline 15 & $84,783,628$ & $85,501,061$ & $\mathrm{LOH}$ \\
\hline 17 & 857,820 & $1,452,131$ & $\mathrm{LOH}$ \\
\hline 18 & $18,535,946$ & $19,513,726$ & $\mathrm{LOH}$ \\
\hline 19 & $41,994,722$ & $48,382,347$ & CN Loss/LOH \\
\hline 21 & $14,414,872$ & $48,129,895$ & CN Loss/LOH \\
\hline
\end{tabular}

$\mathrm{CN}$ copy number, $\mathrm{LOH}$ loss of heterozygosity

both APC and MCC genes, and by implication the intervening gene REEP5 (also known as DP1). On the basis of this additional information, Sanger sequencing of the five exons of REEP5 (NM_005669.4) was performed on the germline DNA of 23 individuals with presumed

Table 3 Regions of loss of heterozygosity $(\mathrm{LOH})$ and copy number (CN) loss in pseudomyxoma peritonei tumour from P2

\begin{tabular}{llll}
\hline Chromosome & Start & End & Event \\
\hline 2 & $241,428,066$ & $243,199,373$ & CN Loss/LOH \\
8 & 0 & $33,395,041$ & CN Loss/LOH \\
8 & $42,137,047$ & $49,106,261$ & LOH \\
10 & 0 & $29,922,641$ & CN Loss \\
11 & $66,296,148$ & $135,006,516$ & LOH \\
11 & $111,333,148$ & $117,815,955$ & CN Loss \\
11 & $130,316,743$ & $135,006,516$ & CN Loss \\
$X$ & 1 & $155,270,560$ & CN Loss/LOH \\
\hline
\end{tabular}

$\mathrm{CN}$, copy number. $\mathrm{LOH}$, loss of heterozygosity 


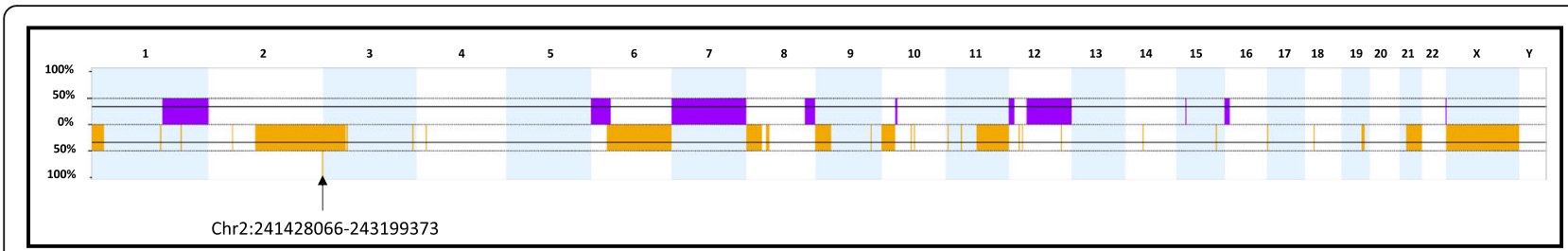

Fig. 2 Summary plot of combined regions of copy number gain (blue) or loss of heterozygosity (brown). Legend. Copy number gain (blue) or loss of heterozygosity (brown), with bars reaching 100\% indicating copy number aberrations seen in both tumours. The region on chromosome 2 containing loss of heterozygosity in both tumours is indicated by the arrow, with genomic coordinates listed below arrow. Chr, chromosome

sporadic AMTs $(\mathrm{n}=13)$ and/or PMP of presumed appendiceal origin (Table 4). The age of diagnosis (extracted from the histopathology report) ranged from 31 to 72 years, with a median age of 58 years. The age for one individual was unknown. No LoF variants or missense variants (excluding common polymorphisms) in REEP5 were identified.

\section{Discussion}

As PMP is a rare disorder, we postulated that this apparent familial occurrence of the disease in a father and daughter might imply a hereditary predisposition. Only a limited assessment of the known colorectal cancer predisposing genes had been performed previously on familial PMP cases in the literature. Through germline whole exome sequencing of both affected individuals in our parent-child PMP family, we were able to assess and exclude the known colorectal cancer predisposing genes including APC, MLH1, MSH2, MSH6, PMS2, MUTYH, BMPR1A, SMAD4, POLE and POLD1, with the caveat

Table 4 Characteristics of the validation cohort of AMTs and/or PMPs

\begin{tabular}{ll}
\hline Characteristic & Number \\
\hline Validation Cases & 23 \\
Male & 12 \\
Female & 11 \\
AMTs & 13 \\
LAMN & 7 \\
Appendiceal mucinous adenocarcinomas & 5 \\
HAMN & 1 \\
Associated with PMP & 10 \\
PMP only & 10 \\
Low grade & 8 \\
High grade & 1 \\
Mixed & 1 \\
Implied history of AMT &
\end{tabular}

AMTs appendiceal mucinous tumours, LAMN low-grade appendiceal mucinous neoplasm, HAMN high grade appendiceal mucinous neoplasm, PMP pseudomyxoma peritonei. \#history of AMT implied in histopathology report that STK11 could not be excluded due to low coverage on exome sequencing.

We took an agnostic approach to identifying the causative gene by identifying novel (ie. not present in the 1000 Genomes Project and NHLBI GO Exome Sequencing Project datasets) shared germline variants in the exome of both individuals, which were also very rare $\left(\leq 1 \times 10^{-4}\right.$ in the gnomAD dataset), coupled with genome-wide copy number analysis of both tumours. We identified 15 potentially pathogenic variants shared between the two cases, including four within regions of $\mathrm{LOH}$ in the father's tumour. Loss of the potentially pathogenic missense variant in $R A N B P 2$ in the tumour genome, suggests this variant is unlikely to be the cause of the PMP predisposition. The missense variants in EXOG, RANBP6 and TNFRSF1B remained heterozygous in the primary tumour of $\mathrm{P} 1$, although with the loss of the second allele at a later stage, this does not necessarily exclude these variants as possible PMP predisposition genes on these data alone.

A shared nonsense variant in REEP5 p.Tyr53* (NM 005669.4:c.159 T > G) is a plausible candidate predisposing variant, although both tumours retained heterozygosity at this locus. REEP5 has been implicated in the regulation of TP53, a known cancer predisposition gene, through its interaction with $\operatorname{HCCR} 1[38,39]$. It is expressed in normal colonic tissue and has been shown to be down-regulated in colon cancers. Similarly, transfection of REEP5 into RKO colon cancer cells results in growth inhibition and induction of apoptosis, suggesting it functions as a tumour suppressor [38]. REEP5 is also involved in stabilisation of the endoplasmic reticulum tubules via its effect on the curvature of the endoplasmic reticulum lipid bilayer [40]. The lack of REEP5 mutations in the 23 validation cases that were screened suggests that germline REEP5 mutations are not common in sporadic AMTs/PMPs, however this is a small dataset of a very rare tumour type, and more cases are required to determine if REEP5 is implicated in the development of AMTs.

Several genes that contained a shared rare missense variant have functions consistent with cancer predisposition. $R H B D L 2$ is a rhomboid intra-membrane protease 
that activates epidermal growth factor and is associated with anoikis resistance [41]. FGFR4 is part of the fibroblast growth factor receptor family, a subset of tyrosine kinase receptors that are highly conserved. The fibroblast growth factor receptors have been extensively investigated somatically in relation to various cancers, and several fibroblast growth factor receptor inhibitors are in clinical trials [42]. The functional consequence of this variant is unknown, however it has a high CADD scaled score and is predicted to be deleterious by SIFT and potentially damaging by PolyPhen, making it an interesting candidate for further functional study. A common germline polymorphism (FGFR4:c.1162G > A) has been associated with an increased risk of developing breast and prostate cancers [43]. EGFR is a receptor tyrosine kinase that is somatically mutated in $10 \%$ of non-small cell lung cancers [44]. Exon 19 deletions and a p.L858R mutation account for approximately $90 \%$ of such mutations, and predict exquisite sensitivity to EGFR tyrosine kinase inhibitors [45-47]. The identified missense variant in this study lies outside the protein kinase domain, hence a potential role in the development of AMTs remains to be determined.

There are a number of limitations with this study. This study only searched exon-based sequences and as such would be unable to identify sequence variants present in gene regulatory regions. The aggregation of PMP within the family may also have arisen due to a shared environmental factor, or due to stochastic events. In view of these limitations, it is possible that the shared variants identified in this study may reflect shared private variants unrelated to a predisposition to AMTs.

The validation cohort consisted of 3 cases ascertained on AMTs without associated PMP on the histopathology report. Conversely for the cases ascertained on PMP alone, an appendiceal primary was not present in the histopathology report, but was alluded to in 4 cases and assumed for the remaining cases. As it is unknown if all AMTs give rise to PMP, further studies of REEP5 in AMTs with known associated PMP may be required to determine if REEP5 mutations predispose to AMTs associated with PMP.

With regards to the use of LOH to identify second hits in candidate genes, PMP is a hypo-cellular tumour with only widely scattered groups of tumour cells in abundant mucin. As there was insufficient DNA in the primary tumours for genome wide copy number analysis, DNA was obtained from associated PMPs. This phenotype provided technical problems in the somatic $\mathrm{LOH}$ analysis.

\section{Conclusions}

Through germline whole exome sequencing of a very rare familial occurrence of a very rare cancer type, in which only 2 families with limited genomic analyses have been reported in the literature, our study makes several contributions to knowledge.

We have identified candidate variants that may predispose to the development of AMTs and PMP in this family. We were also able to examine the known CRCpredisposing genes (apart from STK11) and found no known pathogenic variants in this family, suggesting that other novel genes may predispose to this rare subtype of cancer in the colon. Through the re-sequencing of REEP5 in 23 sporadic AMTs and/or PMP cases, we did not identify any germline REEP5 mutations, despite a shared LoF variant found in our family, and the loss of REEP5 in a case report of a patient with FAP and an appendiceal mucinous tumour. Further studies are required to ascertain if the genes identified in this study play a role in the development of PMP in familial or sporadic cases.

\section{Supplementary information}

Supplementary information accompanies this paper at https://doi.org/10. 1186/s12885-020-6705-y.

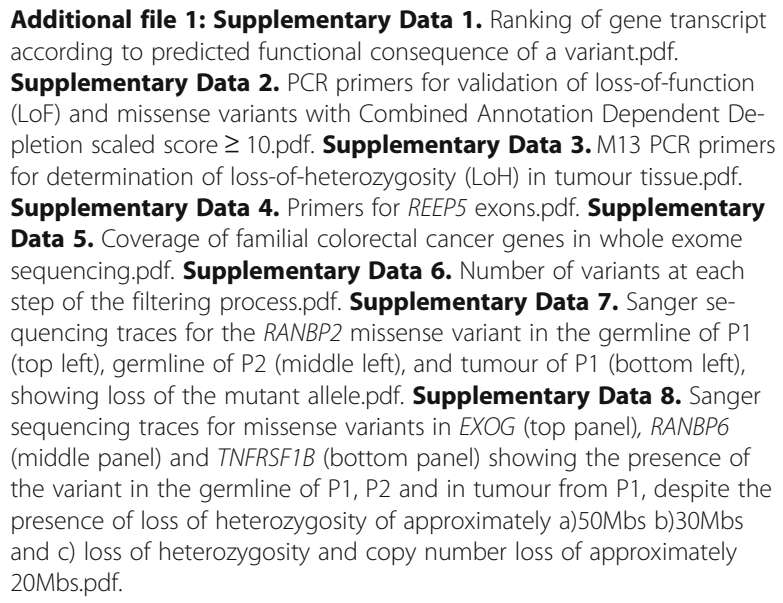

\section{Abbreviations \\ AMT: Appendiceal mucinous tumour; CADD: Combined Annotation Dependent Depletion; DNA: Deoxyribonucleic acid; FAP: Familial adenomatous polyposis; GATK: Genome Analysis Tool Kit; gnomAD: Genome Aggregation Database; HAMN: High grade appendiceal mucinous neoplasm; LAMN: Low-grade appendiceal mucinous neoplasm; LoF: Loss-of-function; \\ LOH: Loss of heterozygosity; NHLBI GO: National Heart, Lung and Blood Institute Grand Opportunity; PCR: Polymerase chain reaction; \\ PMP: Pseudomyxoma peritonei; SNV: Single nucleotide variant}

\section{Acknowledgements}

The Molecular Core facility at Peter MacCallum Cancer Centre performed whole exome sequencing on germline DNA from P1 and P2. Biospecimens used in this research were also obtained from the Victorian Cancer Biobank, Victoria, Australia with appropriate ethics approval. The Victorian Cancer Biobank is supported by the Victorian Government.

Consent to publication

Not applicable. 


\section{Author's contributions}

MSL planned the study, performed whole genome amplification, Sanger sequencing and tumour microdissection, analysed and interpreted the whole exome sequencing data and tumour data and drafted the manuscript. CAM performed the histological examinations of the AMTs and PMP specimens. MAD performed sequence alignment and variant calling. $\mathrm{ACL}$ identified the father and daughter with AMTs. KLG edited the manuscript DDLB is the principal investigator on the Australian Ovarian Cancer Study Group, which provided the sporadic PMP specimens. IGC and AHT conceived and initiated the study, obtained funding, contributed to the interpretation of study data and edited the manuscript. The author(s) read and approved the final manuscript.

\section{Funding}

This study was funded through a grant from the Australian National Health and Medical Research Council and Victorian Cancer Council (APP1028374). Mei Sim Lung is funded by the Australian Postgraduate Award through the University of Melbourne, Australia.

The Australian Ovarian Cancer Study (AOCS) was supported by the U.S. Army Medical Research and Materiel Command under DAMD17-01-1-0729, The Cancer Council Victoria, Queensland Cancer Fund, The Cancer Council New South Wales, The Cancer Council South Australia, The Cancer Foundation of Western Australia, The Cancer Council Tasmania and the National Health and Medical Research Council of Australia (NHMRC; ID400413, ID400281), Ovarian Cancer Australia (OCA) and the Peter MacCallum Foundation.

\section{Availability of data and materials}

The datasets used and/or analysed during the current study are available from the corresponding author on reasonable request.

\section{Ethics approval and consent to participate}

All procedures performed involving human participants were in accordance with the ethical standards of the Peter MacCallum Cancer Centre Human Research and Ethics Committee (project number 10_83) and with the 1964 Helsinki declaration and its later amendments or comparable ethical standards. Written informed consent was obtained from all individual participants included in the study. P1 and P2 signed patient information and consent forms to participate in this study, which included the consent for publication of de-identified data.

\section{Competing interests}

The authors declare that they have no competing interests.

\begin{abstract}
Author details
'Research Division, Cancer Genetics Laboratory, Peter MacCallum Cancer Centre, Victorian Comprehensive Cancer Centre Building, 305 Grattan St., Melbourne, Victoria VIC 3000, Australia. ${ }^{2}$ Department of Pathology, Peter MacCallum Cancer Centre, Melbourne, Victoria, Australia. ${ }^{3}$ Research Computing Facility, Peter MacCallum Cancer Centre, Melbourne, Victoria, Australia. ${ }^{4}$ Department of Surgical Oncology, Peter MacCallum Cancer Centre, Melbourne, Victoria, Australia. ${ }^{5}$ Cancer Genomics Program, Peter MacCallum Cancer Centre, Melbourne, Victoria, Australia. ${ }^{6} \mathrm{Cancer}$ Genetics and Genomics Laboratory, Peter MacCallum Cancer Centre, Melbourne, Victoria, Australia. ${ }^{7}$ Sir Peter MacCallum Department of Oncology, University of Melbourne, Parkville, Victoria, Australia. ${ }^{8}$ Department of Biochemistry and Molecular Biology, University of Melbourne, Parkville, Victoria, Australia. ${ }^{9}$ Parkville Familial Cancer Centre, Peter MacCallum Cancer Centre, Melbourne, Victoria, Australia. ${ }^{10}$ Department of Medicine, University of Melbourne, Parkville, Victoria, Australia.
\end{abstract}

Received: 19 September 2019 Accepted: 2 March 2020 Published online: 01 May 2020

\section{References}

1. Shaib WL, Goodman M, Chen Z, Kim S, Brutcher E, Bekaii-Saab T, et al. Incidence and survival of Appendiceal mucinous neoplasms: a SEER analysis. Am J Clin Oncol. 2017;40(6):569-73.

2. Misdraji J. Mucinous epithelial neoplasms of the appendix and pseudomyxoma peritonei. Mod Pathol. 2015;28(Suppl 1):S67-79.
3. Noguchi R, Yano H, Gohda Y, Suda R, Igari T, Ohta Y, et al. Molecular profiles of high-grade and low-grade pseudomyxoma peritonei. Cancer Med. 2015; 4(12):1809-16.

4. Nummela $P$, Saarinen $L$, Thiel $A$, Jarvinen $P$, Lehtonen $R$, Lepisto $A$, et al. Genomic profile of pseudomyxoma peritonei analyzed using nextgeneration sequencing and immunohistochemistry. Int J Cancer. 2015; 136(5):E282-9.

5. Singhi AD, Davison JM, Choudry HA, Pingpank JF, Ahrendt SA, Holtzman MP, et al. GNAS is frequently mutated in both low-grade and high-grade disseminated appendiceal mucinous neoplasms but does not affect survival. Hum Pathol. 2014;45(8):1737-43.

6. Sio TT, Mansfield AS, Grotz TE, Graham RP, Molina JR, Que FG, et al. Concurrent MCL1 and JUN amplification in pseudomyxoma peritonei: a comprehensive genetic profiling and survival analysis. J Hum Genet. 2014;59(3):124-8.

7. Liu X, Mody K, de Abreu FB, Pipas JM, Peterson JD, Gallagher TL, et al. Molecular profiling of appendiceal epithelial tumors using massively parallel sequencing to identify somatic mutations. Clin Chem. 2014;60(7):1004-11.

8. Alakus H, Babicky ML, Ghosh P, Yost S, Jepsen K, Dai Y, et al. Genome-wide mutational landscape of mucinous carcinomatosis peritonei of appendiceal origin. Genome Med. 2014;6(5):43.

9. Gleeson EM, Feldman R, Mapow BL, Mackovick LT, Ward KM, Morano WF, et al. Appendix-derived Pseudomyxoma Peritonei (PMP): molecular profiling toward treatment of a rare malignancy. Am J Clin Oncol. 2018;41(8):777-83.

10. Pengelly RJ, Rowaiye B, Pickard K, Moran B, Dayal S, Tapper W, et al. Analysis of mutation and loss of Heterozygosity by whole-exome sequencing yields insights into Pseudomyxoma Peritonei. J Mol Diagn. 2018;20(5):635-42.

11. Shih IM, Yan H, Speyrer D, Shmookler BM, Sugarbaker PH, Ronnett BM. Molecular genetic analysis of appendiceal mucinous adenomas in identical twins, including one with pseudomyxoma peritonei. Am J Surg Pathol. 2001;25(8):1095-9.

12. Racek AR, Rabe KG, Wick MJ, Psychogios A, Lindor NM. Primary appendiceal mucinous adenocarcinoma in two first-degree relatives: case report and review. Hered Cancer Clin Pract. 2011;9(1):1.

13. FastQC: a quality control tool for high throughput sequence data. Available at http://www.bioinformatics.babraham.ac.uk/projects/fastqc/.

14. Martin M. Cutadapt removes adapter sequences from high-throughput sequencing reads. EMBnetjournal; Vol 17, No 1: Next Generation Sequencing Data Analysis. 2011.

15. Li H. Aligning sequence reads, clone sequences and assembly contigs with BWA-MEM. 2013 (arXiv:1303.3997v2 [q-bio.GN]).

16. Picard: http://broadinstitute.github.io/picard.

17. McKenna A, Hanna M, Banks E, Sivachenko A, Cibulskis K, Kernytsky A, et al. The genome analysis toolkit: a MapReduce framework for analyzing nextgeneration DNA sequencing data. Genome Res. 2010;20(9):1297-303.

18. McLaren W, Pritchard B, Rios D, Chen Y, Flicek P, Cunningham F. Deriving the consequences of genomic variants with the Ensembl API and SNP effect predictor. Bioinformatics. 2010;26(16):2069-70.

19. Rios D, McLaren WM, Chen Y, Birney E, Stabenau A, Flicek P, et al. A database and API for variation, dense genotyping and resequencing data. BMC Bioinform. 2010;11:238.

20. Genomes Project C, Abecasis GR, Auton A, Brooks LD, DePristo MA, Durbin RM, et al. An integrated map of genetic variation from 1,092 human genomes. Nature. 2012;491(7422):56-65.

21. Exome Variant Server. NHLBI GO Exome Sequencing Project (ESP), Seattle. http://evs.gs.washington.edu/EVS/) n.d. Accessed 19 Nov 2014.

22. Thompson ER, Doyle MA, Ryland GL, Rowley SM, Choong DYH, Tothill RW, et al. Exome Sequencing Identifies Rare Deleterious Mutations in DNA Repair Genes FANCC and BLM as Potential Breast Cancer Susceptibility Alleles. PLoS Genet. 2012;8(9):e1002894.

23. Ramensky V, Bork P, Sunyaev S. Human non-synonymous SNPs: server and survey. Nucleic Acids Res. 2002;30(17):3894-900.

24. Ng PC, Henikoff S. SIFT: predicting amino acid changes that affect protein function. Nucleic Acids Res. 2003;31(13):3812-4.

25. Kircher M, Witten DM, Jain P, O'Roak BJ, Cooper GM, Shendure J. A general framework for estimating the relative pathogenicity of human genetic variants. Nat Genet. 2014;46(3):310-5.

26. Robinson JT, Thorvaldsdottir H, Winckler W, Guttman M, Lander ES, Getz G, et al. Integrative genomics viewer. Nat Biotech. 2011;29(1):24-6.

27. Thorvaldsdóttir H, Robinson JT, Mesirov JP. Integrative genomics viewer (IGV): high-performance genomics data visualization and exploration. Brief Bioinform. 2013;14(2):178-92. 
28. Karczewski KJ, Francioli LC, Tiao G, Cummings BB, Alföldi J, Wang Q, et al. Variation across 141,456 human exomes and genomes reveals the spectrum of loss-of-function intolerance across human protein-coding genes. 2019:531210.

29. Untergasser A, Cutcutache I, Koressaar T, Ye J, Faircloth BC, Remm M, et al. Primer3--new capabilities and interfaces. Nucleic Acids Res. 2012;40(15):e115.

30. Koressaar T, Remm M. Enhancements and modifications of primer design program Primer3. Bioinformatics. 2007;23(10):1289-91.

31. Thomas NA, Neville PJ, Baxter SW, Campbell IG. Genetic analysis of benign ovarian tumors. Int J Cancer. 2003;105(4):499-505.

32. Kempers MJ, Kuiper RP, Ockeloen CW, Chappuis PO, Hutter P, Rahner N, et al. Risk of colorectal and endometrial cancers in EPCAM deletion-positive Lynch syndrome: a cohort study. Lancet Oncol. 2011;12(1):49-55.

33. Palles $\mathrm{C}$, Cazier JB, Howarth KM, Domingo E, Jones AM, Broderick P, et al. Germline mutations affecting the proofreading domains of POLE and POLD1 predispose to colorectal adenomas and carcinomas. Nat Genet. 2013;45(2):136-44

34. de Voer RM, Geurts van Kessel A, Weren RD, Ligtenberg MJ, Smeets D, Fu L, et al. Germline mutations in the spindle assembly checkpoint genes BUB1 and BUB3 are risk factors for colorectal cancer. Gastroenterology. 2013; 145(3):544-7.

35. Rio Frio T, Lavoie J, Hamel N, Geyer FC, Kushner YB, Novak DJ, et al. Homozygous BUB1B mutation and susceptibility to gastrointestinal neoplasia. N Engl J Med. 2010;363(27):2628-37.

36. Weren RD, Ligtenberg MJ, Kets CM, de Voer RM, Verwiel ET, Spruijt L, et al. A germline homozygous mutation in the base-excision repair gene NTHL1 causes adenomatous polyposis and colorectal cancer. Nat Genet. 2015;47(6): 668-71.

37. Koorey D, Basha NJ, Tomaras C, Freiman J, Robson L, Smith A. Appendiceal carcinoma complicating adenomatous polyposis in a young woman with a de novo constitutional reciprocal translocation t (5;8)(q22;p23.1). J Med Genet. 2000;37(1):71-5.

38. Shin SM, Chung YJ, Oh ST, Jeon HM, Hwang L, Namkoong H, et al. HCCR1-interacting molecule "deleted in polyposis 1" plays a tumor-suppressor role in colon carcinogenesis. Gastroenterology. 2006;130(7):2074-86.

39. Ko J, Lee YH, Hwang SY, Lee YS, Shin SM, Hwang JH, et al. Identification and differential expression of novel human cervical cancer oncogene HCCR-2 in human cancers and its involvement in p53 stabilization. Oncogene. 2003; 22(30):4679-89.

40. Burke B. PREEParing for mitosis. Dev Cell. 2013;26(3):221-2.

41. Cheng T-L, Lai C-H, Jiang S-J, Hung J-H, Liu S-K, Chang B-I, et al. RHBDL2 is a critical membrane protease for Anoikis resistance in human malignant epithelial cells. Sci World J. 2014;2014:8.

42. Katoh M, Nakagama H. FGF receptors: Cancer biology and therapeutics. Med Res Rev. 2014;34(2):280-300

43. $X u$ W, Li Y, Wang $X$, Chen B, Wang Y, Liu S, et al. FGFR4 transmembrane domain polymorphism and cancer risk: a meta-analysis including 8555 subjects. Eur J Cancer. 2010;46(18):3332-8.

44. Lynch TJ, Bell DW, Sordella R, Gurubhagavatula S, Okimoto RA, Brannigan $\mathrm{BW}$, et al. Activating mutations in the epidermal growth factor receptor underlying responsiveness of non-small-cell lung cancer to gefitinib. N Engl J Med. 2004;350(21):2129-39.

45. Rosell R, Carcereny E, Gervais R, Vergnenegre A, Massuti B, Felip E, et al. Erlotinib versus standard chemotherapy as first-line treatment for European patients with advanced EGFR mutation-positive non-small-cell lung cancer (EURTAC): a multicentre, open-label, randomised phase 3 trial. Lancet Oncol. 2012;13(3):239-46.

46. Zhou C, Wu YL, Chen G, Feng J, Liu XQ, Wang C, et al. Erlotinib versus chemotherapy as first-line treatment for patients with advanced EGFR mutation-positive non-small-cell lung cancer (OPTIMAL, CTONG-0802): a multicentre, open-label, randomised, phase 3 study. Lancet Oncol. 2011; 12(8):735-42.

47. Mok TS, Wu Y-L, Thongprasert S, Yang C-H, Chu D-T, Saijo N, et al. Gefitinib or carboplatin-paclitaxel in pulmonary adenocarcinoma. N Engl J Med. 2009;361(10):947-57.

\section{Publisher's Note}

Springer Nature remains neutral with regard to jurisdictional claims in published maps and institutional affiliations.

\section{Ready to submit your research? Choose BMC and benefit from}

- fast, convenient online submission

- thorough peer review by experienced researchers in your field

- rapid publication on acceptance

- support for research data, including large and complex data types

- gold Open Access which fosters wider collaboration and increased citations

- maximum visibility for your research: over $100 \mathrm{M}$ website views per year

At $\mathrm{BMC}$, research is always in progress.

Learn more biomedcentral.com/submissions 